\title{
Liquid crystal cell with a tapered optical fiber as an active element to optical applications
}

\author{
Joanna E. Moś, Karol A. Stasiewicz, and Leszek R. Jaroszewicz \\ Faculty of New Technology and Chemistry, Military University of Technology, gen. Sylwestra Kaliskiego \\ Received February 14, 2019; accepted March 31, 2019; published March 31, 2019
}

\begin{abstract}
The work describes the technology of a liquid crystal cell with a tapered optical fiber as an element providing light. The tapered optical fiber with a total optical loss of $(0.22 \pm 0.07) \mathrm{dB}$, taper waist diameter of $(15.5 \pm 0.5) \mu \mathrm{m}$, and elongation of $(20.4 \pm 0.3) \mathrm{mm}$ has been used. The experimental results are presented for a liquid crystal cell filled with the mixture $1550^{*}$ for parallel orientation of LC molecules to the cross section of a taper waist. Measurement results show the influence of an electrical field with voltage in the range of $0 \div 200 \mathrm{~V}$, without/with different modulation for spectral characteristics. Sinusoidal and square signal shapes are used with a $1 \div 10 \mathrm{~Hz}$ frequency range.
\end{abstract}

Fiber optic technology is widely used in telecommunications, as well as in measurement applications. Currently, various fiber optic techniques are used to obtain optical sensors with higher accuracy parameters such as sensitivity, detection speed, etc. One of the possibilities is the connection of an optical fiber with additional material as an active medium. Usually, microstructured fibers with additional material inside of the fiber are used for non-telecommunications application of optic fiber [1], for example by filling them with a liquid material $[2 \div 3]$. There is also a possibility of using extra material outside the fiber as a deposited layer to protect interactions with the environment [4]. Because optical fibers are designed to keep the light inside the core and not interact with an external environment, a special method for structure modification, especially the boundary condition, should be used. In these methods, the phenomena of an evanescent field will be used $[5 \div 6]$. There are various technological solutions including: bending the optical fiber [7], polishing or etching the cladding $[9 \div 10]$, or using a tapered optical fiber $[8 \div 13]$. The latter technique can be used for any kind of fiber like standard single mode SMF, multimode optical fiber MMF, photonics and others.

Tapered optical fiber technology allows us to manufacture different devices by using additional materials. Such materials can be also treated as active elements of an optical system. In many cases their properties like absorption, reflection allow detecting even the smallest changes in measuring physical, biological or chemical properties $[14 \div 15]$. The use of a tapered optical fiber has also many advantages: its manufacturing

*E-mail: joanna.musial@wat.edu.pl technology is already well known and controlled, in-line elements - produced, compactness achieved up to nanometres, combination a tapered optical fiber possible with many types of materials like metals [16 $\div 17]$, alkanes [18], materials like gelatine [19], metal oxides [11, 20], polymer layers [12], etc. Appropriate optimization of tapered optical fiber parameters like elongation length, diameter and length of a taper waist, taper geometry offer the possibility of using tapered optical fiber in sensors application.

The material used in this work is a mixture of liquid crystals (LCs). Its anisotropic properties and ability to control its optical properties through voltage and temperature allowed us to apply it as a cladding material around the taper waist. An external electric field allows for reorientation of molecules in an LC layer and change of a medium refractive index (director of LC molecules) from ordinary $n_{\mathrm{o}}$ to extraordinary $n_{\mathrm{e}}$ value. Another factor which allows for changing the refractive index value is temperature. In this case, the LC mixture changes the value of a refractive index from initial value located between $n_{\mathrm{o}}$ and $n_{\mathrm{e}}$ to the value of isotropic liquid $n_{\text {iso }}$ after exceeding appropriate $T_{\text {iso }}$ temperature. These changes are related to phase changes of the liquid crystal from nematic to isotropic phase [23]. In this way, the combination of tapered fiber optic technology and liquid crystal properties allows us to create a hybrid connection which works as an active optical element enabling to control the properties of a propagating light beam. As a result, the possibility of creating optical filters or sensors is obtained $[24 \div 26]$. Similar devices were produced earlier [27] but controlling parameters was possible only for laboratory use. The obtained parameter for presented LC cells - a much lower electric field - shows potential external application, however the parameters such as threshold voltage and switching times should be improved in the future, mainly by the reduction of cell thickness and application of a tapered optical fiber with optimized parameters.

One of the most important steps of this work is the technology of the LC cell with a tapered optical fiber. The cell was built entirely in FOTET (Fiber Optic Taper Element Technology). This device allows for fabrication of a tapered optical fiber, by heating a small section of the 
fiber to a softening point of glass and by pulling out fiber by stepper motors. The important advantage of FOTET is the possibility to influence the length of a taper waist (area with the smallest diameter) [26]. It can be controlled by three torch movement modes: lack of movement, constant movement, and increasing movement. The longest taper waist is obtained in constant movement mode. In our case, 5-mm-movement of a torch was applied. This is important due to the interplay between material length and propagating beam. The tapered optical fiber was characterized for the following parameters: loss of $(0.22 \pm 0.07) \mathrm{dB}, \quad(15.5 \pm 0.5) \mu \mathrm{m}$ diameter of taper waist and elongation length equal to $(20.3 \pm 0.5) \mathrm{mm}$. The cell consists of plates with an ITO (indium-tin oxide) layer and a layer orienting the molecules. The research presented in the article is obtained for a cell with a parallel orientation to the cross section of the taper waist.

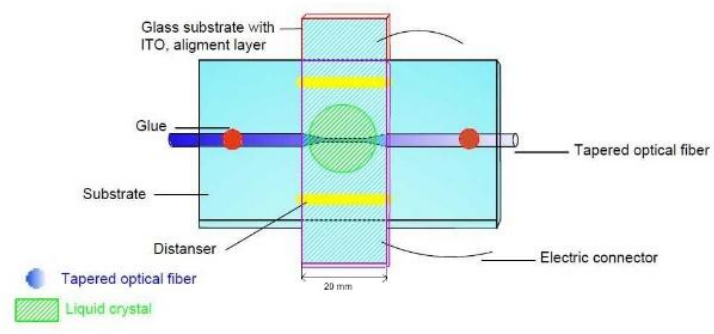

Fig. 1. Scheme of the LC cell with a tapered optical fiber.

The cell under investigation was filled with liquid crystalline mixture $1550^{*}$ characterized by the following parameters: birefringence $\Delta n=0.068, \quad n_{0}=1.4618$, $n_{\mathrm{e}}=1.5276$ (for a $589 \mathrm{~nm}$ wavelength and temperature equal to $20^{\circ} \mathrm{C}$ ), and the temperature of transition into isotropic state $T_{i s o}=79.8^{\circ} \mathrm{C}$ [28]. The LC mixture was synthesized at the Institute of Chemistry at MUT. The thickness of the cell is $40 \mu \mathrm{m}$ determined by used spacers.

When we consider a single-mode fiber where the fundamental mode is propagated in the structure, in the beginning, the light propagates as a mode strongly trapped in the core. Theoretically, it is described by a total internal reflection. As a result of tapering the fiber structure, the fiber diameter decreases and the difference between the refractive index of the core and the cladding is not sufficient to maintain the mode in the core. During the technological process, the geometry of fiber is changed so that the light does not see the boundary condition between the core and the cladding. The light expands in the cladding and is propagated as a cladding mode and interacts with the LC mixture $[29 \div 30]$. In the study, the tapered optical fiber is surrounding an LC material which at room temperature has a higher reflective index of the optical fiber cladding. By increasing the temperature, $n_{\mathrm{o}}$ and $n_{\mathrm{e}}$ values of LC become decreased. Over $35^{\circ} \mathrm{C}$, the refractive index $n_{\mathrm{o}}$ of
LC is lower than the refractive index of a fiber optic cladding (1.46) [28]. If the refractive index of the optical fiber taper is higher than the cladding, then the light is propagated using the law of total internal reflection, otherwise in the LC some part of power is propagating in the region of the cladding as shown Fig. 2.

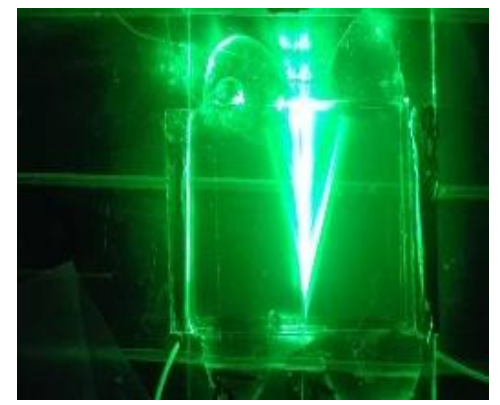

Fig. 2. LC cell with a tapered optical fiber. Light is propagated in the LC cladding.

Applied electrical field with a suitable amplitude can effectively reorient LC molecules and allow light propagation for a given wavelength as shown in Fig. 3.

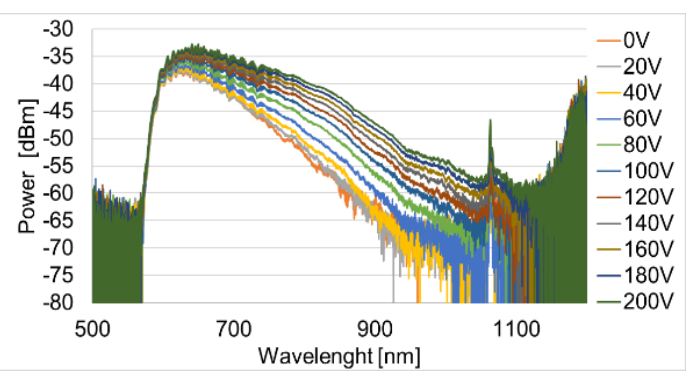

Fig. 3. Spectral characteristics in the wavelength range of $500 \div 1200 \mathrm{~nm}$ for the LC cell with a tapered optical fiber for different applied voltage at room temperature.

As one can see, the spectral characteristics changes are observed in the wavelength range of $600 \div 1100 \mathrm{~nm}$ for a different electric field applied. For other wavelengths, the transmission is completely attenuated by the used LC mixture. Observed increased transmission with increased voltage is due to a change in the orientation of LC molecules which are aligned with the direction of the electric field. The refractive index of LC changes from $n_{\mathrm{e}}$ to $\mathrm{n}_{\mathrm{o}}$ value in this situation and if a given condition (mainly temperature) is lower than the tapered optical fiber, the light is effectively transmitted by the structure.

The influence of electrical signal modulation (its shape and frequency) on spectral characteristics can be observed only after exceeding the applied voltage of $80 \mathrm{~V}$. This value is strictly connected with cell thickness. The shape, as well as frequency of a driven signal, is mapped on the transmission spectrum and observed as amplification of some ranges of wavelengths depending on applied frequency. The results presented in Figs. 4 and 5 indicate that sinusoidal, as well as square shapes, are mapped for different frequencies in the range of $1 \div 10 \mathrm{~Hz}$. In general, 
the modulation depth in spectral characteristics depends on frequency and increases with frequency.

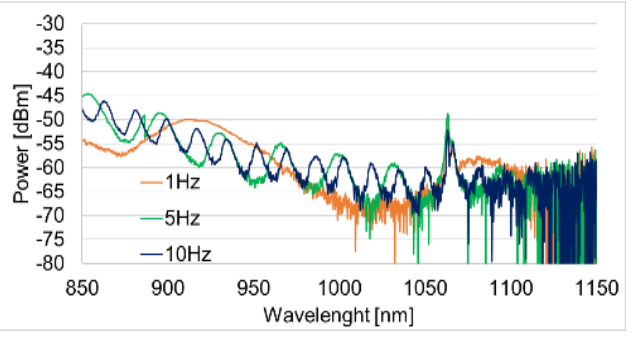

Fig. 4. Spectral characteristics in the wavelength range of $850 \div 1150 \mathrm{~nm}$ for applied $160 \mathrm{~V}$ signal amplitude. The sinusoidal shape of modulation with a frequency of $1 \div 10 \mathrm{~Hz}$ has been applied.

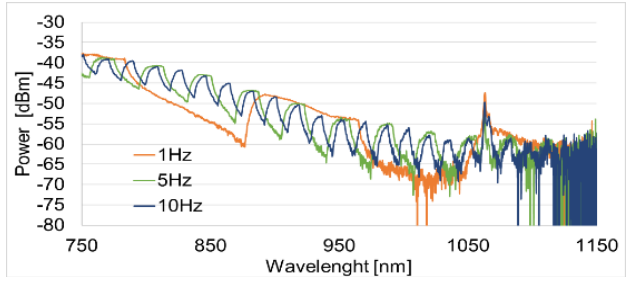

Fig. 5. Spectral characteristics in the wavelength range of $850 \div 1150 \mathrm{~nm}$ for applied $160 \mathrm{~V}$ signal amplitude. The square shape of modulation with a frequency of $1 \div 10 \mathrm{~Hz}$ has been applied.

For higher frequency, the square signal shape is not well mapped as one can see in Fig. 5 for $10 \mathrm{~Hz}$. The spectral response depends on the speed of molecules switching and associated relaxation time where the molecules should return to their initial state of alignment (Fig. 6). The time of relaxation depends on the thickness of the cell, as well as the properties of used LC mixture. As it is well known, the used $1550^{*}$ mixture is generally a very slow nematic liquid crystal.

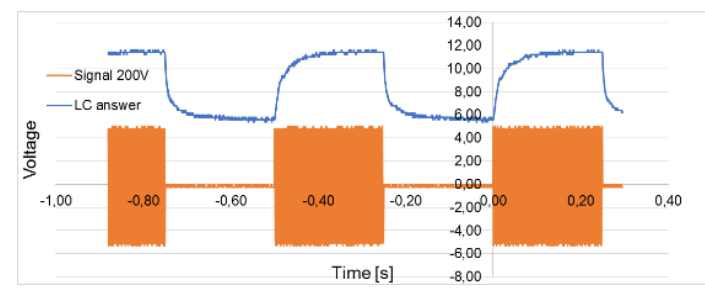

Fig. 6. Time course of the LC cell with a tapered optical fiber for an applied electric field of $200 \mathrm{~V}$ with $2 \mathrm{~Hz}$ modulation.

In conclusions, the hybrid combination of fiber optic and liquid crystal technologies gives us the opportunity to create an active element that allows controlling the properties of a propagating light beam in a fiber. The application of a suitable electrical field gives the possibility to control the transmission characteristics of the presented structure. In addition, electrical signal modulation causes changes in the spectral characteristics, where the shape of the signal and frequency changes are shown. The obtained results can be used as the basis for construction of optical devices such as filter or optical sensors. The next milestone in the research will be improving cell parameters. Cell thickness will be reduced by applying a suitable optical fiber taper structure and smaller spacers. Such an approach should effectively reduce the voltage amplitude of a steering signal. Additionally, we are going to apply another material - a mixture of liquid crystal and nanoparticles. These technological changes will ensure reduced threshold voltage and relaxation time.

This article was prepared with the support of National Science Centre of Poland PRELUDIUM 2018/29/N/ST7/02347 and RMN 08/689 from Military University of Technology

\section{References}

[1] Z. Liu, H. Y. Tam, L. Htein, M. L.Vincent Tse, C. Lu J. Lightwave Technol. 35, 16 (2017).

[2] T.R. Wolinski, K. Szaniawska, S. Ertman, P. Lesiak, A.W. Domański, R. Dabrowski, E. Nowinowski-Kruszelnicki, J. Wojcik Meas. Sci. Technol. 17, 5 (2006).

[3] K. Nielsen, D. Noordegraaf, T. Sørensen, A. Bjarklev,T. Hansen, J. Opt. A: Pure Appl. Opt. 7, 8 (2005).

[4] A.A. Rifat, G.A. Mahdiraji, D.M. Chow, Y. Gang Shee, R. Ahmed, F. Rafiq, M Adikan, Sensors 15, 5 (2015).

[5] Y. Huang, Z.Tian, L.P. Sun, D. Sun, J.Li, Y.Ran, B.-O. Guan, Opt. Expr. 23, 21 (2015)

[6] X. Wang, O.S. Wolfbeis Anal. Chem., 88, 1 (2016).

[7] Ye Tian, W. Wang, N. Wu, X. Zou, X.Wang Sensors 11, 4 (2011).

[8] O. Katsunari, Fundamentals of Optical Waveguides (London, Academic Press (2006).

[9] A.K. Sharma, J. Rajan, B.D. Gupta IEEE Sensors Journal 7, 8 (2007).

[10] C. Caucheteur, T. Guo, J. Albert Anal. Bioanal.Chem. 407, 14 (2015).

[11] S.F. Silva L. Coelho, O. Frazão, J.L. Santos, F.X.r Malcata, IEEE Sensors Journal 12, 1 (2012).

[12] H. Waechter, J. Litman, A.H. Cheung, J.A. Barnes, H.P. Loock Sensors 10, 3 (2010).

[13] S. Zhu, F. Pang, S. Huang, F. Zou, Y. Dong, T.Wang Opt. Expr. 23, 11 (2015)

[14] L. Zhang, J. Lou, L. Tong, Photonics Sensor 1, 1 (2011)

[15] L.Tong, J. Lou, E. Mazur, Opt. Expr. 11, 6 (2004).

[16] H. Moyyed, I.T. Leite, L. Coelho, J.L. Santos, D. Viegas IEEE Sensors Journal 14, 10 (2014).

[17] A. González-Cano, M. Cruz Navarette, Ó. Esteban, N. Diaz Herrera Sensors 14, 3 (2014)

[18] K.A. Stasiewicz, J.E. Moś Opt. Fiber Technol. 32, (2016).

[19] L. Zhang, F. Gu, J. Lou, X. Yin, L. Tong Opt. Expr. 16, 17 (2008).

[20] S.Zhu, F.Pang, S. Huang, F. Zou, Q. Guo, J. Wen, T. Wang, Sensors 16, 8 (2016).

[21] G.Brambilla, J. Optics 12, 4 (2010).

[22] M. Ahmad, L.L. Hench Biosens. Bioelectron. 20, 7 (2005).

[23] L.M. Blinov, Electrooptic Effects in Liquid Crystal Materials (New York, Springftianer, 1994).

[24] L. Scolari, T.T. Alkeskjold, A. Bjarklev Electron. Lett. 42, 22 (2006).

[25] J. Moś, M. Florek, K. Garbat, K.A. Stasiewicz, N. Bennis, L.R. Jaroszewicz, J. Lightwave Technol. 36, 4 (2017).

[26] J. Moś, K.A. Stasiewicz, K. Garbat, P. Morawiak, W. Piecek, L.R. Jaroszewicz, Phys. Scripta 93, 12 (2018).

[27] Ch. Veilleux, J. Lapierre, J. Bures, Opt. Lett. 11, 11 (1986).

[28] R. Dąbrowski, K. Garbat, S. Urban, T.R. Woliński, J. Dziaduszek, T. Ogrodnik, A.Siarkowska Liq. Cryst. 44, 1911 (2017).

[29] S. Lacroix, R.J. Black, Ch. Veilleux, J. Lapierre Opt. Soc.of Am. 25, 15 (1986).

[30] J.F. Henninot, D. Louvergneaux, N.Tabiryan, M. Warenghem, Mol. Cryst Liq.Cryst. 282, 1 (1996). 